\title{
Long-Term Efficacy and Safety of Atazanavir/Ritonavir Treatment in a Real-Life Cohort of Treatment-Experienced Patients with HIV Type 1 Infection
}

\author{
Klaus Jansen, Anders Sönnerborg, ${ }^{2}$ Norbert Brockmeyer, ${ }^{3}$ Anders Thalme, ${ }^{2}$ \\ Veronica Svedhem, ${ }^{2}$ Stephan Dupke, ${ }^{4}$ Jean-Luc Eychenne, ${ }^{5}$ Tina Nakonz, \\ Maria Jesus Jimenez-Exposito, ${ }^{7}$ and Pascal Pugliese ${ }^{8}$
}

\begin{abstract}
Atazanavir-based regimens have established efficacy and safety in both antiretroviral (ARV)-naive and -experienced patients. However, data evaluating effectiveness beyond 2 years is sparse. Therefore, we assessed the long-term outcomes of ritonavir-boosted atazanavir (ATV/r)-containing regimens in ARV-experienced patients in a clinical setting in a noncomparative, retrospective, observational study collecting data from three European HIV databases on ARV-experienced adults with HIV-1 infection starting an ATV/r-based regimen. Data were extracted every 6 months (maximum follow-up 5 years). Primary outcome was the proportion of patients remaining on ATV/r by baseline HIV-1 RNA ( $<500$ or $\geq 500$ copies $/ \mathrm{ml}$ ). Secondary outcomes included time to virologic failure, reasons for discontinuation, and long-term safety profile. The duration of treatment and time to virologic failure were analyzed using the Kaplan-Meier method. Data were analyzed for 1,294 ARV-experienced patients (male 74\%; mean ART exposure 5.7 years). After 3 years, 56\% (95\% CI: 52\%, 60\%) of patients with baseline HIV-1 RNA < 500 copies/ml and 53\% (95\% CI: 49\%, 58\%) of those with HIV-1 RNA $\geq 500$ copies $/ \mathrm{ml}$ remained on ATV $/ \mathrm{r}$. After 3 years, 75\% (95\% CI: 69\%, 80\%) of patients with baseline HIV-1 RNA < 50 copies $/ \mathrm{ml}$ remained suppressed and 51\% (95\% CI: 47\%, 55\%) of those with baseline HIV-1 RNA $\geq 50$ copies/ml achieved and maintained virologic suppression. Although adverse events (AEs) were the main known reason for discontinuation, no unexpected AEs were observed. In a real-life setting ATV/r-based regimens demonstrated sustained virologic suppression in ARV-experienced patients. After long-term therapy the majority of patients remained on treatment and no unexpected AEs were observed.
\end{abstract}

\section{Introduction}

$S^{n}$ NCE THEIR INTRODUCTION IN 1996, it is estimated that combined antiretroviral therapy (cART) has halved the average mortality rate for HIV-1-infected individuals in the developed world. ${ }^{1}$ As a result, long-term treatment outcomes and regimen durability have become more clinically relevant.

Despite these improvements in CART, many patients change treatment for reasons that may include virologic failure, adverse events, or poor adherence consequent to pill burden or tolerability issues. Therefore, the long-term out- comes of patients receiving different antiretroviral (ARV) regimens, both as initial therapy and after switching, are essential to evaluate given the longer life expectancy associated with modern cART. ${ }^{1}$

Although randomized clinical trials are generally conducted over a substantial duration, typically 96 weeks, cohort studies of an even longer duration have the potential to offer valuable additional information with respect to long-term outcomes of therapy in a real-life clinical setting.

The introduction of protease inhibitors (PIs) into HIV treatment combinations has made a significant contribution

\footnotetext{
${ }^{1}$ Competence Network for HIV / AIDS, Ruhr-Universität, Bochum, Germany.

${ }^{2}$ Karolinska Institute, Stockholm, Sweden.

${ }^{3}$ St. Josef-Klinik, Ruhr-Universität, Bochum, Germany.

${ }^{4}$ Clinical Centre Driesener Straße, Berlin, Germany.

${ }^{5}$ Hays Pharma, Paris, France.

${ }^{6}$ Bristol-Myers Squibb, Munich, Germany.

${ }^{7}$ Bristol-Myers Squibb, Paris, France.

${ }^{8}$ Service d'Infectiologie, Nice University Hospital, Nice, France.
} 
to improvements in morbidity and mortality. Several PI agents are now available, providing options for individualized therapy, and most regimens are boosted with the pharmacological enhancer, ritonavir. ${ }^{2}$

In clinical trials, convenient once-daily ritonavir-boosted atazanavir (ATV / r)-based regimens have proven efficacy and safety in both treatment-naive ${ }^{3-5}$ and -experienced patients. ${ }^{6,7}$ These regimens are recommended as a preferred therapeutic option for initiation of therapy in treatment-naive patients by the current treatment guidelines in both the United States ${ }^{8,9}$ and Europe. ${ }^{10}$

In clinical settings, ATV/r-based regimens are associated with sustained virologic suppression and favorable rates of treatment discontinuation when compared with other ARV regimens. ${ }^{11-14}$ However, information about these long-term real-life outcomes is sparse in patients receiving ATV $/ \mathrm{r}^{11-13}$ and in patients receiving boosted PI-based therapy in general, ${ }^{15}$ especially in the case of treatment-experienced patients. ${ }^{16,17}$

Therefore, the aim of the current study was to evaluate the long-term outcomes of ATV/r-based regimens in a large sample of ARV-experienced patients in a real-life European clinical setting. To our knowledge this represents the largest cohort to date evaluating long-term outcomes of a boosted PI-based regimen in ARV-experienced patients.

\section{Materials and Methods}

\section{Study design}

This was a noncomparative, retrospective, observational study of ARV-experienced patients (including PI-experienced) stratified by viral load at baseline (HIV-1 RNA < 500 copies / $\mathrm{ml}$ or $\geq 500$ copies $/ \mathrm{ml}$ ). Data were collected from three generic HIV databases: two multicenter databases, one based in France (Dat'AIDS) ${ }^{18}$ and the other in Germany (Competence Network for HIV / AIDS, KompNet), ${ }^{19}$ and one single-center database based in Sweden (InfCare HIV). Patients were initiated on an ATV/r-based regimen between October 1, 2004 and March 31, 2007.

HIV patient databases were identified in several European countries: France, Germany, Sweden, Italy, Switzerland, United Kingdom, Denmark, and Spain. After conducting a feasibility study to assess the output and access of each database, the general information and exposure data collected for each patient, and the compatibility of the type of data collected with the requirements of the study protocol, three databases were finally selected from France, Germany, and Sweden.

An integrated study dataset of patients switching to an ATV / r-based regimen was then generated from these three generic datasets and patient data were extracted at 6-monthly intervals until October 31, 2009 (maximum follow-up period of 5 years).

Although most variables were collected and reported in a similar way across all three databases, some variables were collected and reported differently between databases. These variables included baseline Centers for Disease Control (CDC) AIDS class, baseline hepatitis coinfection, mode of HIV acquisition, and reasons for treatment discontinuation. To consistently analyze these variables, they were mapped in the study dataset across the three individual databases and grouped into those categories commonly used in clinical trials and cohort studies.

The study was conducted in accordance with the ethical principles originating from the Declaration of Helsinki, and was consistent with the International Conference on Harmonization Good Clinical Practice Guidelines, Guidelines for Good Pharmacoepidemiology Practices, and local regulatory requirements. Approval for cohort inclusion was obtained from the local ethical committees of all study centers.

\section{Patients}

Two patient populations were studied, stratified according to viral load at baseline: those with a threshold viral load of either HIV-1 RNA $<500$ or $\geq 500$ copies $/ \mathrm{ml}$. In the absence of a universally agreed definition of virologic failure across the three individual databases, we chose to use the 500 copies $/ \mathrm{ml}$ cut-off value because it was the most commonly used, both in clinical practice at the time and within the databases themselves. Therefore, the 500 copies $/ \mathrm{ml}$ cut-off value gave the best available classification of patients who were virologically suppressed or not at baseline after switching to an ATV/rbased regimen. However, given that current clinical practice would now favor a definition of virologic failure based upon a lower cut-off value, results for the analysis of time to virologic failure were also reported stratifying patients using the more recent and stringent lower limit of quantification (LLOQ) cutoff value of 50 copies $/ \mathrm{ml}$ (baseline threshold viral load either HIV-1 RNA $<50$ or $\geq 50$ copies $/ \mathrm{ml}$ ).

Inclusion criteria were participation in a European HIV cohort, age $\geq 18$ years on commencement of ATV/r therapy, ARV experience prior to starting ATV / r therapy, and ATV $/ \mathrm{r}$ therapy commenced between October 1, 2004 and March 31, 2007. Patients were excluded if they were treatment naive or had no recorded start date for ATV/r therapy.

\section{Outcomes}

The primary outcome was the proportion of patients remaining on treatment over time, stratified by plasma viral load at baseline (i.e., HIV-1 RNA $<500$ copies/ml or $\geq 500$ copies/ml at study entry). The probability of remaining on treatment over time was estimated using the Kaplan-Meier method.

Secondary outcomes included proportions of patients discontinuing ATV/r treatment over time, reasons for treatment discontinuation, time to virologic failure estimated using the Kaplan-Meier method, change in CD4 counts estimated using mixed models repeated measures (MMRM) analysis, and the long-term safety profile of ATV/r treatment.

Virologic failure was defined using the most stringent LLOQ of 50 copies $/ \mathrm{ml}$ and was recorded as having occurred following either two consecutive HIV-1 RNA $\geq 50$ copies $/ \mathrm{ml}$ tests or one HIV-1 RNA $\geq 50$ copies/ml test followed by discontinuation. Virologic failure was also analyzed using the LLOQ of 500 copies/ml. When using the LLOQ of 50 copies $/ \mathrm{ml}$ definition of virologic failure, it was considered more appropriate to stratify the results by the same cut-off value of 50 copies $/ \mathrm{ml}$ at baseline in order to evaluate patients using the same criterion before and after ATV/r treatment. Similarly, when using the definition of LLOQ of 500 copies/ml, stratification by 500 copies $/ \mathrm{ml}$ at baseline was employed.

\section{Statistical analyses}

For the analysis of time-to-event data, the Kaplan-Meier method was considered to be the most appropriate technique 
and a strategy for handling censored observations designed to minimize the overestimation of treatment effects was employed. Thus, all patients were analyzed up to the date of their last contact, including patients with ATV/r treatment interruptions, provided the duration of interruption was $\leq 90$ days. Patients with missing data or who were lost to followup were censored.

For the analysis of CD4 counts, MMRM was considered to be a more appropriate method for imputing missing data than the last observation carried forward (LOCF) method because MMRM generates estimates that are valid for most types of missing data, whereas LOCF does not. ${ }^{20}$

The long-term safety profile of $\mathrm{ATV} / \mathrm{r}$ treatment was evaluated by collecting data on reported adverse events and laboratory abnormalities. Adverse event data were collected and analyzed for all patients up to the point of ATV $/ r$ treatment discontinuation or until the end of the follow-up period.

Multivariate Cox proportional hazards regression analyses were used to determine factors associated with ATV $/ \mathrm{r}$ treatment discontinuation and virologic failure. Gender and baseline viral load, variables known to be associated with these outcomes, were forced into the model. Additional covariates were entered if they demonstrated $\leq 20 \%$ of missing values, absence of colinearity, and a $p$-value of $<0.20$ on a prescreening univariate analysis. Variables entered into the preliminary univariate analysis included age, country, mode of HIV acquisition, baseline CD4 cell count, and previous exposure to ARV therapy (drug class and duration).

\section{Results}

Patients

A total of 1,294 patients were included in the study and were followed up for a total of 2,748 patient-years. Details of patient disposition are summarized in Fig. 1. Baseline characteristics overall, by baseline viral load, and by country were generally similar and are presented in Table 1 . The median age in the overall cohort at ATV/r initiation was 43 years, and $74 \%$ were men ( $85 \%$ in the German cohort). Approximately one-third of participants were classified with CDC Class C AIDS. About $75 \%$ of patients had prior experience of PI therapy, and the mean duration of previous ARV experience was over 5 years.

\section{Primary outcome}

The probability of remaining on ATV/r-based regimen is shown in Fig. 2a (Kaplan-Meier analysis). After 3 years of treatment, $56 \%$ (95\% CI: $52 \%, 60 \%)$ of patients with baseline virologic suppression (<500 copies $/ \mathrm{ml})$ and 53\% (95\% CI: $49 \%, 58 \%$ ) of those with detectable viremia ( $\geq 500$ copies $/ \mathrm{ml}$ ) remained on treatment.

\section{Secondary outcomes}

Treatment discontinuation. Overall, the median time to discontinuation was estimated to be 45 months (95\% CI: 41, 49) for the overall cohort, with slightly longer median duration of therapy (48 months; $95 \%$ CI: 10,52$)$ for those patients with baseline virologic suppression, relative to those with baseline detectable viremia (44 months; 95\% CI: 36, 50).

Of 1,294 patients overall, 43.1\% (558) discontinued ATV/r treatment over time. Discontinuation rates in patients with a baseline HIV-1 RNA level of $<500$ copies $/ \mathrm{ml}$ or $\geq 500$ copies $/ \mathrm{ml}$ were $41.6 \%$ and $45.2 \%$, respectively. The proportion of patients discontinuing $\mathrm{ATV} / \mathrm{r}$ treatment was $21.2 \%$ up to the first year, $32.7 \%$ up to the second year, $39.8 \%$ up to the third year, $42.6 \%$ up to the fourth year, and $43.1 \%$ up to 60 months (missing data excluded).

Adverse events (11\%), patient decision (6\%), and lack of efficacy $(5 \%)$ were among the most common documented

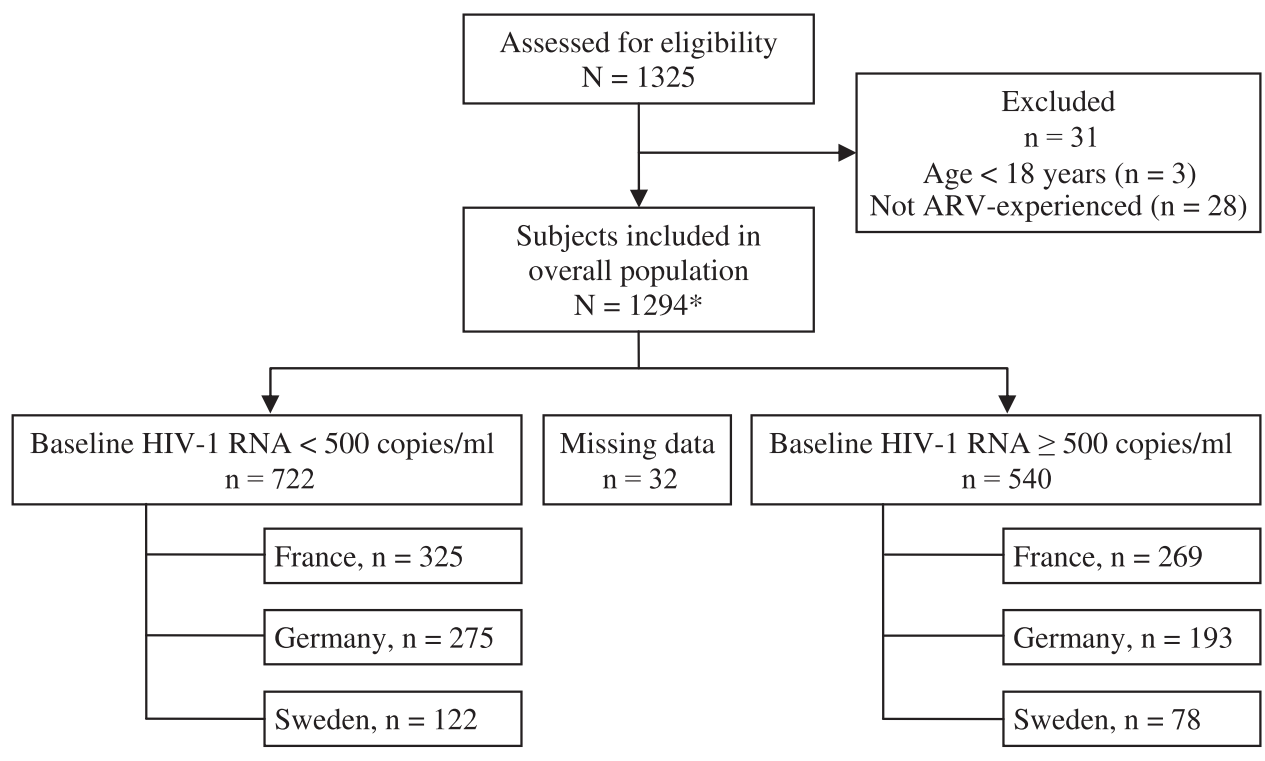

*1130 patients with baseline HIV-1 RNA assessment using an assay precision of at least 50 copies $/ \mathrm{ml}$

- Baseline HIV-1 RNA level < 50 copies/ml $(\mathrm{n}=413)$

- Baseline HIV-1 RNA level $\geq 50$ copies/ml $(n=717)$

FIG. 1. Patient disposition. 
Table 1. Baseline Characteristics by Plasma HiV-1 RNA Level and Country

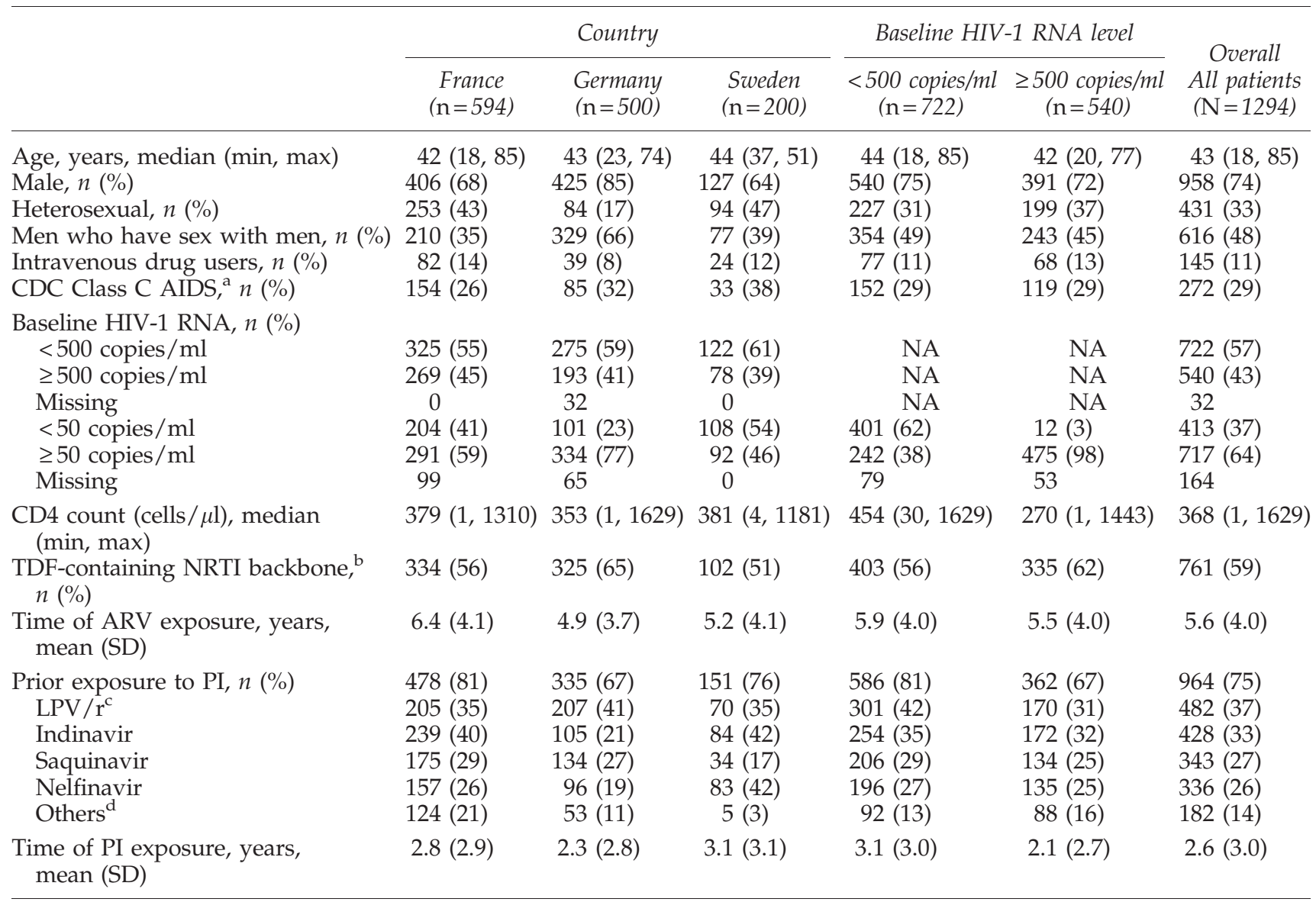

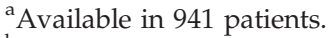

${ }^{\mathrm{b}}$ Available in 1287 patients.

${ }^{\mathrm{c}}$ Available in 1280 patients.

${ }^{\mathrm{d}}$ Includes fosamprenavir ( $6 \%$ of patients overall), unboosted atazanavir $(4 \%)$, amprenavir $(3 \%)$, tipranavir $(1 \%)$, and darunavir $(0.1 \%)$.

NA, not applicable; CDC, Centers for Disease Control; TDF, tenofovir disoproxil fumarate; LPV/r, ritonavir-boosted lopinavir; PI, protease inhibitor; NRTI, nucleoside reverse transcriptase inhibitor; ARV, antiretroviral.
}

reasons for treatment discontinuation. Lack of efficacy was defined as treatment failure (clinical, virologic, or immunologic), resistance, drug interaction, or other therapeutic reasons. The reason for discontinuation was unknown in $14 \%$ of patients.

\section{Time to virologic failure}

After 3 years of treatment, 91\% (95\% CI: 87\%, 93\%) of patients $(n=722)$ with baseline HIV-1 RNA <500 copies $/ \mathrm{ml}$ remained suppressed, and 65\% (95\% CI: 60\%,69\%) of patients ( $n=540$ ) with baseline HIV-1 RNA $\geq 500$ copies/ml achieved virologic suppression and remained suppressed. Using the more stringent definition of virologic failure, after 3 years of treatment, $75 \%$ (95\% CI: $69 \%, 80 \%)$ of patients $(n=413)$ with baseline HIV-1 RNA <50 copies/ml remained suppressed, and $51 \%$ (95\% CI: $47 \%, 55 \%)$ of patients $(n=717)$ with baseline HIV-1 RNA $\geq 50$ copies/ml achieved virologic suppression and remained suppressed (Fig. 2b).

\section{CD4 cell count analysis}

CD4 cell counts increased progressively over the entire study period; the estimated mean CD4 count increase for all patients was 48 cells / $\mu \mathrm{l} /$ year (95\% CI: 45, 50; $p<0.0001$ ) (Fig. 3). Increases were most rapid for patients with baseline detectable viremia (HIV-1 RNA $\geq 500$ copies $/ \mathrm{ml}$ ) who had an estimated mean CD4 count increase of 61 cells/ $\mu$ l/year (95\% CI: 58, 65; $p<0.0001$ ). Corresponding values for patients with baseline HIV-1 RNA $<500$ copies $/ \mathrm{ml}$ were 38 cells / $\mu \mathrm{l}$ /year (95\% CI: 35, 41; $p<0.0001$ ).

\section{Adverse events}

Adverse events, regardless of causality, are presented in Table 2. Hyperbilirubinemia occurred in $63 \%$ of patients at any time point. However, jaundice and discontinuation due to hyperbilirubinemia were infrequent, occurring in 12 patients $(<1 \%)$ irrespective of baseline HIV-1 RNA levels. Nephrolithiasis occurred in 7 patients $(<1 \%)$.

\section{Multivariate analysis}

Female gender (hazard ratio 1.54; 95\% CI: 1.28, 1.85; $p<0.001$ ), but not baseline detectable viremia HIV-1 RNA $\geq 500$ copies $/ \mathrm{ml}$ (hazard ratio $1.04 ; 95 \%$ CI: 0.88, 1.24; $p=0.630$ ), was significantly associated with an increased risk of $\mathrm{ATV} / \mathrm{r}$ treatment discontinuation.

Baseline detectable viremia HIV-1 RNA $\geq 500$ copies $/ \mathrm{ml}$ (hazard ratio $2.93 ; 95 \%$ CI: 2.36, 3.64; $p<0.001$ ) and baseline 

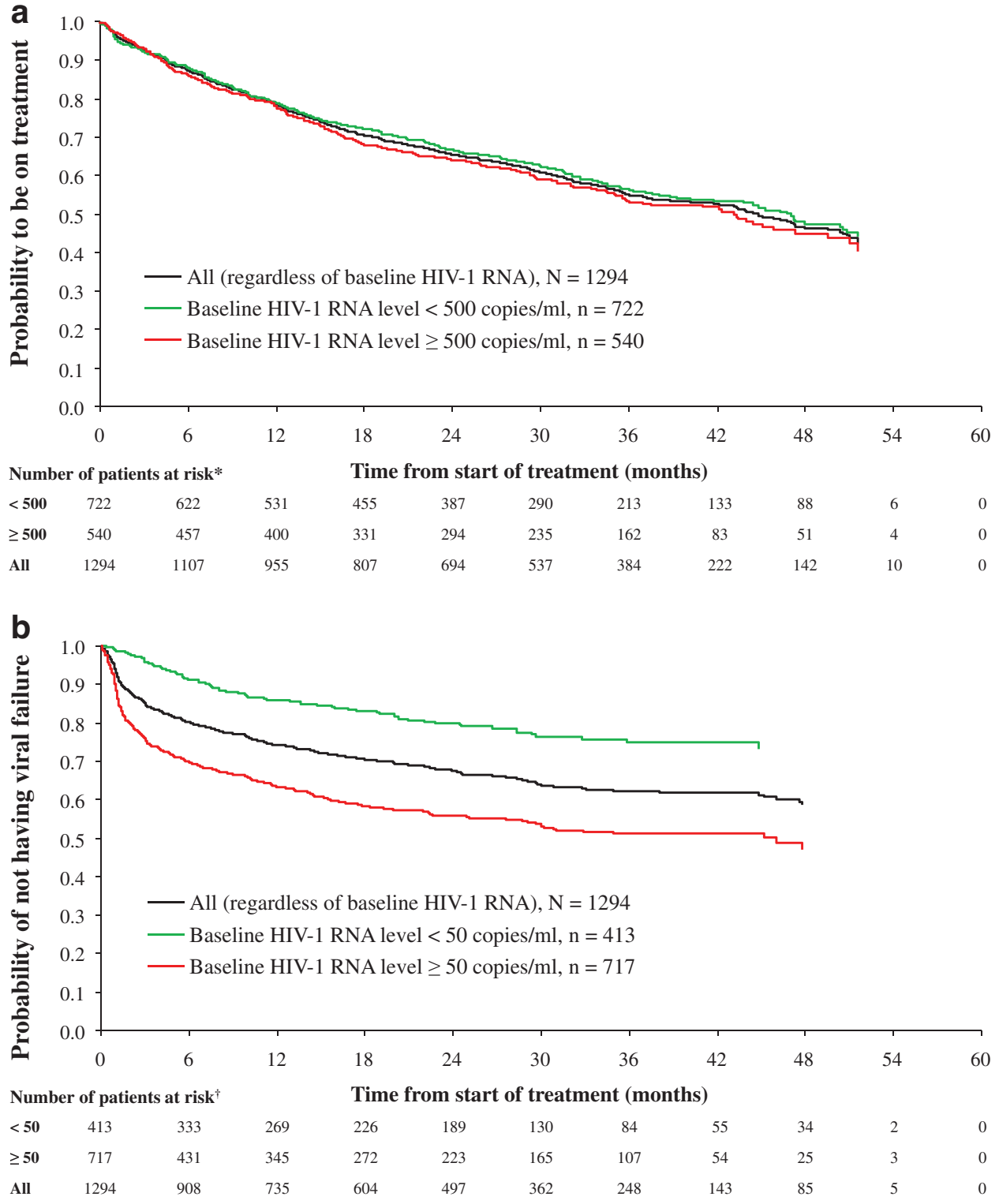

FIG. 2. Kaplan-Meier survival function. (a) Time to discontinuation and (b) time to virologic failure. $N$ is the number of patients at baseline. There were $* 32$ patients who had a missing baseline HIV-1 RNA assessment using an assay precision of at least 500 copies $/ \mathrm{ml}$ and 164 patients who had a missing baseline HIV-1 RNA assessment using an assay precision of at least 50 copies $/ \mathrm{ml}$; thus, the total number of patients at risk irrespective of baseline HIV-1 RNA status at each time point is greater than the corresponding sum of patients at risk with available baseline HIV-1 RNA assessments.
CD4 cell count (hazard ratio 1.56; 95\% CI: 1.26, 1.98; $p<0.001$ ), but not female gender (hazard ratio 1.06; 95\% CI: 0.85, 1.33; $p=0.612$ ), were significantly associated with an increased risk of virologic failure.

Country (Sweden versus France) and type of last concomitant therapy (that is the last drug class recorded in combination with $\mathrm{ATV} / \mathrm{r}$ before discontinuation) were also associated with treatment discontinuation and virologic failure in the main-effects model (Table 3). However, significant interactions between these and other variables in the full main-effects plus interactions model did not permit meaningful interpretation of country and last concomitant therapy results (Supplementary Table S1; Supplementary Data are available online at www.liebertpub.com/aid).

\section{Discussion}

Compared with randomized clinical trials where patients are selected, long-term cohort studies are more reflective of routine care, and therefore provide an important opportunity to translate clinical trial efficacy to clinical effectiveness in the routine care setting. ${ }^{21}$ Thus, data from randomized clinical trials and cohort studies are collectively important to inform treatment decisions. However, the relative lack of long-term data limits the development of treatment guidelines with respect to recommendations for long-term treatment decisions, especially options for optimizing switch strategies. ${ }^{8,10,22}$ In this retrospective, observational, real-life study examining longterm outcomes in treatment-experienced patients who were switched to an ATV/r-based regimen according to two clinical scenarios, either stable virologic suppression or virologic failure, new data are provided on virologic efficacy, safety, and treatment durability for up to a 5-year period of follow-up.

Virologic suppression was achieved and maintained among a substantial proportion of patients with detectable viremia at baseline, and was maintained among those already virologically suppressed at the time of switch to an ATV/rbased regimen. Virologic response was associated with a 


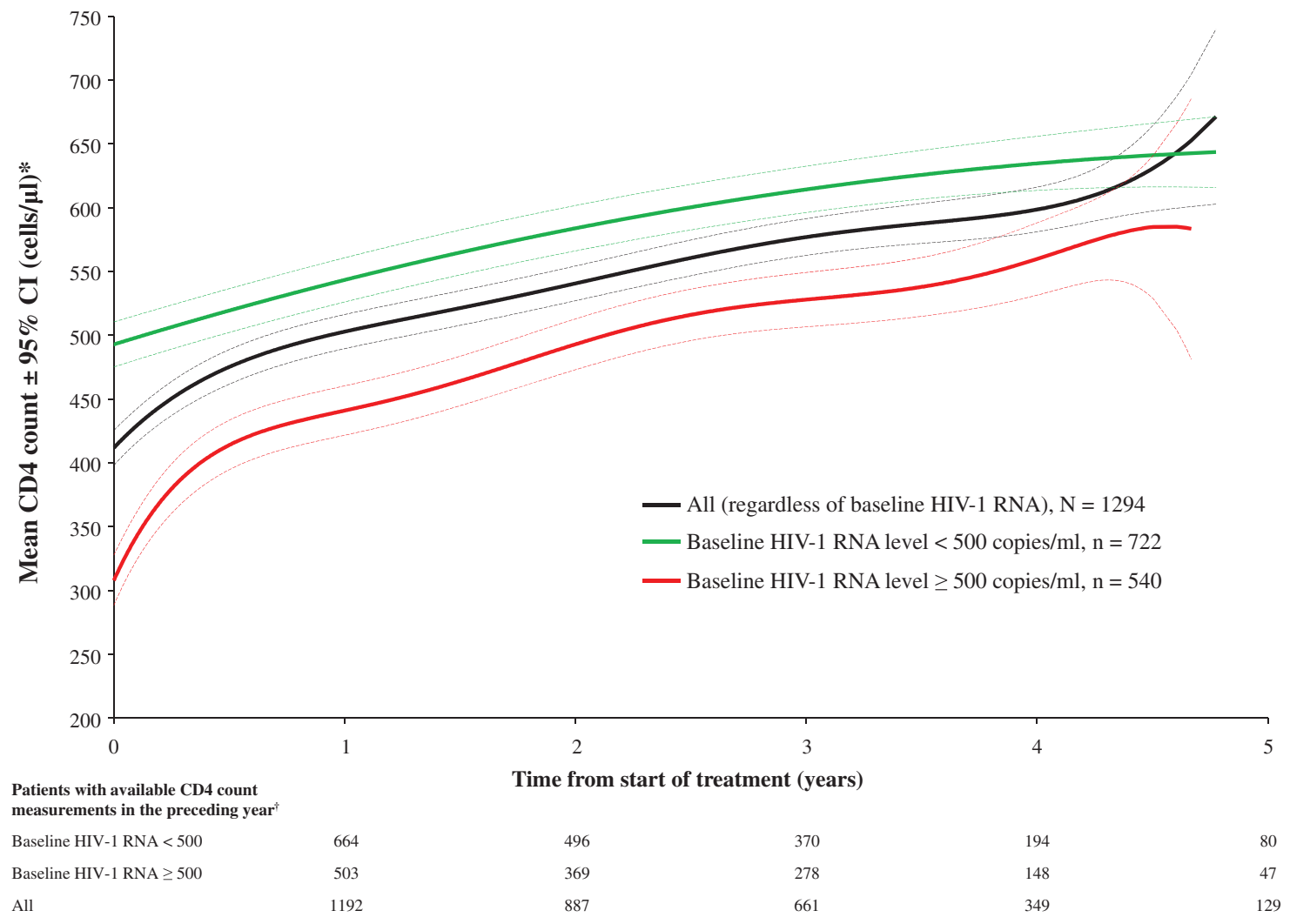

FIG. 3. Mean CD4 counts over time. $N$ is the number of patients at baseline. *Data are predicted mean CD4 counts (solid lines) $\pm 95 \%$ confidence intervals (CI; dotted lines) derived from a mixed models repeated measures analysis. ${ }^{\dagger}$ There were 32 patients who had a missing baseline HIV-1 RNA assessment using an assay precision of at least 500 copies/ml; thus, the total number of patients with available CD4 count measurements irrespective of baseline HIV-1 RNA status at each time point is greater than the corresponding sum of patients with available CD4 count measurements and baseline HIV-1 RNA assessments.

sustained immunological recovery over time. ${ }^{6,7}$ These longterm outcomes confirm and extend the efficacy findings from ATV/r clinical trials in treatment-experienced patients. 6,7

$\mathrm{ATV} / \mathrm{r}$-based regimens were generally well tolerated with a favorable rate of discontinuation over time when compared with other cohort studies examining discontinuation rates with several different first-line ARV regimens. ${ }^{11,12}$ For example, among the seven first-line ARV regimens compared in the Swiss HIV Cohort Study, treatment modification rates at 1 year were lowest for tenofovir-emtricitabine combined with ATV /r (24.1\%) and highest for zidovudine-lamivudine combined with ritonavir-boosted lopinavir $(70.7 \%) .{ }^{11}$ These rates are consistent with the treatment discontinuation rate up to 1 year of $21.2 \%$ found in the current study, which notably evaluated a much larger number of treatment-experienced patients switched to ATV/r. When examining longer-term data, the durability of second-line ATV/r-based regimens in the current study is consistent with findings from a comparative 5-year cohort study of persistence on first-line cART according to the third agent employed in which median persistence on therapy was greater for ATV/r (1,016 days) compared with efavirenz (974 days) or ritonavir-boosted lopinavir (382 days). ${ }^{12}$ Directly comparing treatment-naive and -experienced cohorts can be problematic, but it is important to place these data into the available context given that comparative cohort data on treatment-experienced patients are extremely limited both for patients receiving ATV/r-based regimens ${ }^{23,24}$ and for patients receiving other ARV regimens in general. Despite the difficulty in making comparisons, the fact that the rate of discontinuation with ATV/r in the current study was lower than that observed in other studies using other ARV regimens is relevant from a clinical perspective, especially considering that treatment options for treatmentexperienced patients (particularly those with a viral load $>500$ copies $/ \mathrm{ml}$ at baseline) are limited.

As noted in previous studies, ${ }^{11,25}$ gender differences in discontinuation rate, but not in virologic failure rate, were also observed in the present study. Although further investigations are required to better characterize differences in discontinuation rates, factors such as pregnancy and differences in adherence $e^{25,26}$ or safety profile $\mathrm{e}^{11,26}$ between sexes have been proposed as potential reasons to explain the increased rate of discontinuation observed in females.

The reason for discontinuation was unknown in $14 \%$ of patients, which was not surprising in the context of a cohort study in which such data may not routinely be collected as would be expected in a clinical trial setting. While adverse events were the main known reason for discontinuation, the long-term safety profile in this real-life study was consistent with that observed previously in clinical trials of up to 2 years duration, ${ }^{5-7}$ with no new or unexpected adverse events identified.

Hyperbilirubinemia is associated with ATV treatment, an effect that is considered to be mediated by the inhibition of 
Table 2. Selected Adverse Events (Regardless of Causality)

\begin{tabular}{|c|c|c|c|}
\hline & Basel & $N A^{\mathrm{a}}$ & \\
\hline & $\begin{array}{c}<500 \text { copies } / \mathrm{ml} \\
(\mathrm{n}=722)\end{array}$ & $\begin{array}{c}\geq 500 \text { copies } / \mathrm{ml} \\
(\mathrm{n}=540)\end{array}$ & $\begin{array}{l}\text { All patients } \\
(\mathrm{N}=1294)\end{array}$ \\
\hline Clinical adverse events (any grade), $n(\%)$ & & & \\
\hline Nausea & $3(<1)$ & $4(<1)$ & $7(<1)$ \\
\hline Diarrhea & $30(4)$ & $20(4)$ & $52(4)$ \\
\hline Jaundice & $3(<1)$ & $4(<1)$ & $7(<1)$ \\
\hline Renal and urinary disorders & $22(3)$ & $15(3)$ & $37(3)$ \\
\hline Laboratory adverse events (grade $3-4), n^{\mathrm{b}}$ & & & \\
\hline Total cholesterol ( $\geq 300 \mathrm{mg} / \mathrm{dl})$ & $39 / 505(8)$ & 20/391 (5) & $60 / 924(7)$ \\
\hline Triglycerides $(\geq 751 \mathrm{mg} / \mathrm{dl})$ & $16 / 503(3)$ & $21 / 388(7)$ & $37 / 919(4)$ \\
\hline LDL-cholesterol ( $\geq 190 \mathrm{mg} / \mathrm{dl})$ & $38 / 386(10)$ & $17 / 258(7)$ & $57 / 669(9)$ \\
\hline Total bilirubin elevation $(>2.5 \times \mathrm{ULN})$ & $323 / 512(63)$ & $230 / 401(57)$ & $573 / 939(61)$ \\
\hline Creatinine $(>2 \times U L N)$ & $7 / 302(2)$ & $6 / 242(3)$ & $13 / 544(2)$ \\
\hline Adverse events leading to discontinuation, & & & \\
\hline Patients with adverse events & $82(11)$ & $55(10)$ & $138(11)$ \\
\hline Side effects (total of those listed below) & $15(2)$ & $17(3)$ & $33(3)$ \\
\hline Cutaneous & $13(2)$ & $8(1)$ & $21(2)$ \\
\hline Gastrointestinal & $8(1)$ & $7(1)$ & $15(1)$ \\
\hline Liver/pancreas toxicity & $6(<1)$ & $2(<1)$ & $8(<1)$ \\
\hline Renal & $5(<1)$ & $2(<1)$ & $7(<1)$ \\
\hline Hyperbilirubinemia & $7(<1)$ & $5(<1)$ & $12(<1)$ \\
\hline Metabolic & $6(<1)$ & $0(0)$ & $6(<1)$ \\
\hline Lipodystrophy & $7(<1)$ & $4(<1)$ & $11(<1)$ \\
\hline Other & $15(2)$ & $10(2)$ & $25(2)$ \\
\hline
\end{tabular}

${ }^{a}$ Thirty-two patients with missing baseline HIV-1 RNA assessment using an assay precision of at least $500 \mathrm{copies} / \mathrm{ml}$.

${ }^{b}$ Patients with at least one laboratory value above thresholds while on treatment.

${ }^{\mathrm{c}}$ Patients with laboratory parameter values while on treatment. Toxicity grades were defined according to the Division of AIDS Table for Grading the Severity of Adult and Pediatric Adverse Events.

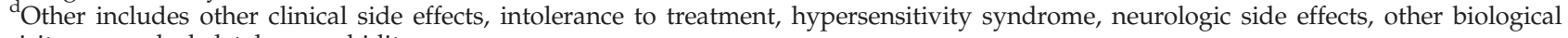
toxicity, musculoskeletal, comorbidity.

LDL, low-density lipoprotein; ULN, upper limit of normal.

uridine 5'-diphosphoglucuronosyltransferase, the enzyme responsible for glucuronidation of bilirubin. ${ }^{27}$ Although ATVinduced hyperbilirubinemia is manageable, reversible, and independent of hepatocellular toxicity, the related symptoms of jaundice and ocular icterus could potentially have an impact on a patient's appearance and quality of life (QoL). ${ }^{28}$ As expected with an ATV/r-based regimen, grade 3-4 hyperbilirubinemia occurred in $63 \%$ of patients in this study at any time point. However, jaundice and discontinuation due to hyperbilirubinemia were rare, occurring in $<1 \%$ of patients. These findings are consistent with those of the CASTLE study, in which hyperbilirubinemia led to discontinuation in $<1 \%$ of patients without impact on adherence or QoL. ${ }^{28}$

Cases of nephrolithiasis have been reported in association with PIs, including ATV $/ \mathrm{r}^{29}$ While data from a recent retrospective study suggested a higher rate of nephrolithiasis with $\mathrm{ATV} / \mathrm{r}^{30}$ methodologic limitations of this study require additional investigations to be undertaken to further explore any potential association of specific drugs with nephrolithiasis. In the present study, nephrolithiasis was rarely reported ( $<1 \%$ of patients).

Owing to the retrospective, cohort design of this study, a number of limitations must be considered. First, the specific reasons for switching to $\mathrm{ATV} / \mathrm{r}$ were not collected in the current study. Although it is reasonable to assume that patients with detectable viral load at baseline $(41.7 \%)$ were switched to ATV/r due to virologic failure, in those patients with undetectable viral load (58.3\%) the reasons for switching to $\mathrm{ATV} / \mathrm{r}$ may be varied, for example, regimen simplification or drug toxicity. In addition, although it was possible to identify a historical list of the specific drugs administered prior to switching to ATV $/ r$, the combination of drugs taken (i.e., the regimen) could not be ascertained with certainty from the available cohort data. Second, bias from missing data or patients lost to follow-up cannot be excluded. Third, in contrast to randomized controlled trials in which all adverse events are systematically reported and assessed for causality, in real-life cohort studies minor adverse events may not be routinely reported and causality is difficult to establish. Thus, although adverse events in the current cohort could have been underreported, adverse events that were unrelated to ATV /r treatment may also have been reported. In addition, adverse events reported in the context of long-term cohort studies may be more reflective of clinical practice and therefore provide additional clinically relevant information complementary to that obtained from randomized controlled trials. Finally, information on genotypic resistance was not available for this study making it difficult to interpret reasons for virologic failure.

Despite these potential limitations, study strengths were the inclusion of a large number of patients from different European countries who were followed up over an extended period. In addition, the lack of narrow selection criteria, as commonly occurs in clinical trials, allowed for an 
Table 3. Multivariate Cox Proportional Hazards Model: ATV/r Treatment Discontinuation and Time to Virologic Failure

\begin{tabular}{|c|c|c|c|}
\hline Covariate $^{\mathrm{a}}$ & Hazard ratio ${ }^{\mathrm{b}}$ & $95 \% \mathrm{CI}$ & $\mathrm{p}$-value \\
\hline \multicolumn{4}{|l|}{$\mathrm{ATV} / \mathrm{r}$ treatment discontinuation } \\
\hline Gender: females vs. males & 1.54 & $1.28,1.85$ & $<0.001$ \\
\hline Baseline detectable viremia $\geq 500$ copies $/ \mathrm{ml}$, yes vs. no & 1.04 & $0.88,1.24$ & 0.630 \\
\hline Germany vs. France & 1.17 & $0.96,1.42$ & 0.129 \\
\hline Sweden vs. France & 1.39 & $1.11,1.75$ & 0.004 \\
\hline Protease inhibitors vs. no protease inhibitors ${ }^{c}$ & 3.21 & $2.67,3.86$ & $<0.001$ \\
\hline NRT inhibitors vs. no NRT inhibitors ${ }^{c}$ & 1.43 & $1.05,1.95$ & 0.023 \\
\hline Non-NRT inhibitors vs. no non-NRT inhibitors ${ }^{c}$ & 2.22 & $1.76,2.80$ & $<0.001$ \\
\hline Other antiretrovirals vs. no other antiretrovirals ${ }^{c}$ & 1.77 & $1.01,3.13$ & $<0.048$ \\
\hline \multicolumn{4}{|l|}{ Time to virologic failure } \\
\hline Gender: females vs. males & 1.06 & $0.85,1.33$ & 0.612 \\
\hline Baseline detectable viremia $\geq 500$ copies $/ \mathrm{ml}$, yes vs. no & 2.93 & $2.36,3.64$ & $<0.001$ \\
\hline Germany vs. France & 0.95 & $0.76,1.20$ & 0.674 \\
\hline Sweden vs. France & 1.63 & $1.25,2.13$ & $<0.001$ \\
\hline Protease inhibitors vs. no protease inhibitors ${ }^{c}$ & 1.96 & $1.56,2.47$ & $<0.001$ \\
\hline NRT inhibitors vs. no NRT inhibitors ${ }^{c}$ & 1.97 & $1.27,3.07$ & 0.003 \\
\hline Non-NRT inhibitors vs. no non-NRT inhibitors ${ }^{c}$ & 1.51 & $1.09,2.11$ & 0.014 \\
\hline Baseline CD 4 count, $<200$ cells $/ \mu$ l vs. $\geq 200$ cells $/ \mu 1$ & 1.56 & $1.26,1.98$ & $<0.001$ \\
\hline
\end{tabular}

${ }^{a}$ Number (\%) of patients used in the model, $1262(98 \%)$.

${ }^{\mathrm{b}}$ Covariate adjusted hazard ratio of time to discontinuation or virologic failure. Only significant variables $(p$-value $<0.05)$ were included with the exception of gender and detectable viremia, which were known to be significant and so have been forced into the model. The model was rerun with only those variables kept in the final model using backward elimination, thus maximizing the number of patients used in the analysis.

'Use as the last concomitant therapy (that is the last class of treatment recorded in combination with ATV/r before discontinuation).

CI, confidence interval; NRT, nucleoside reverse transcriptase.

assessment of the long-term outcomes of ATV/r-based regimens in a real-life clinical setting. Thus, cohort studies provide complementary information on patients who would otherwise have been ineligible for participation in randomized controlled trials. ${ }^{31}$ Moreover, carefully designed cohort studies evaluated in tandem can yield results consistent with those from randomized controlled trials. ${ }^{32,33}$

In this real-life ARV-experienced cohort over a follow-up period of up to 5 years, ATV/r-containing regimens resulted in sustained virologic suppression with a favorable rate of discontinuation over time when compared to other cohort studies, and a long-term safety profile consistent with that previously observed in clinical trials. Thus, ATV/r-containing regimens can be considered as an alternative treatment strategy among ARV-experienced patients who need to switch therapy.

\section{Acknowledgments}

This study was supported by Bristol-Myers Squibb, the manufacturers of atazanavir. Data analyses and clinical study report writing were performed by the contract research organization PRA International and funded by Bristol-Myers Squibb. The results and the clinical study report were reviewed and validated by the Bristol-Myers Squibb project team. All authors directed the scientific content and contributed to the drafting and critical evaluation of the manuscript. The authors thank Jackie Campbell and Julian Martins of inScience Communications, Springer Healthcare who provided medical writing support funded by Bristol-Myers Squibb.

The following documenting sites contributed data to the Dat'AIDS cohort: Unité de Virologie Clinique, CHU de Nice; Service des Maladies Infectieuses, CHU de Toulouse, Service des Maladies Infectieuses, CHU de Fort de France; Service des
Maladies Infectieuses, Hôpital Pitié-Salpétrière, Paris, Service des Maladies Infectieuses Hôpital Dron, Tourcoing, Service des Maladies Infectieuses, CHU de Nantes; Service d'Immuno-Hématologie Clinique Hôpital Sainte-Marguerite APH Marseille. The following documenting sites contributed data to the KompNet cohort: Medical Practice Driesener Straße, Berlin; Medical Practice Mehringdamm, Berlin; Medical Practice Turmstraße, Berlin; Medical Practice Fuggerstraße, Berlin; Medical Practice Kaiserdamm, Berlin; University Clinic Benjamin Franklin, Charité, Berlin; Clinic for Dermatology, Ruhr-Universität, Bochum; Clinic Dortmund; University Clinic, Düsseldorf; Medical Clinic 3, University Clinic, Erlangen; Clinic for Dermatology, Universität Essen; HIVCENTER, University Clinic, Frankfurt; Ifi-Institut, Hamburg; ICH Grindelpraxis, Infektionsepidemiologisches Centrum, Hamburg; Medizinische Hochschule, Hannover; Medical Practice Georgstraße, Hannover; Medical Practice, Kriegsstraße, Karlsruhe; Clinic Kemperhof, Koblenz; Medical Practice Hohenstaufenring, Köln; Medical Practice Isartorplatz, München; MVZ Karlsplatz, HIV Research and Clinical Centre, München; Medical Practice Franz Joseph-Straße, München; Clinic, Osnabrück; Medical Practice Ulmer/ Frietsch/Müller, Stuttgart. The following documenting sites contributed data to the InfCare HIV cohort: Karolinska University Hospital, Stockholm.

\section{Author Disclosure Statement}

K.J. has served on speakers' bureaus and has received honoraria from Bristol-Myers Squibb. A.S. has served on advisory boards and/or speakers' bureaus and has received honoraria or consulting fees from Abbott, Bristol-Myers Squibb, Gilead, GlaxoSmithKline, Jansen-Cilag, and Pfizer. 
N.B. has served on advisory boards and/or speakers' bureaus and has received honoraria or consulting fees from Abbot and Essex Pharma. A.T. has served on advisory boards and/or speakers' bureaus and has received honoraria or consulting fees from Bristol-Myers Squibb, Gilead, GlaxoSmithKline, and Merck Sharp \& Dohme. V.S. has served on advisory boards and/or speakers' bureaus and has received honoraria or consulting fees from Abbott, Bristol-Myers Squibb, GlaxoSmithKline, Merck Sharp \& Dohme, and Roche. J-L.E. is an employee of Hays Pharma and provided statistical support to the study, which was funded by Bristol-Myers Squibb. M.J.J-E. and T.N. are employees of Bristol-Myers Squibb. P.P. has received honoraria for advisory board membership from Bristol-Myers Squibb, Gilead, and Merck Sharp \& Dohme.

\section{References}

1. Ray M, Logan R, Sterne JA, et al.: The effect of combined antiretroviral therapy on the overall mortality of HIVinfected individuals. AIDS 2010;24:123-137.

2. Naggie $S$ and Hicks C: Protease inhibitor-based antiretroviral therapy in treatment-naive HIV-1-infected patients: The evidence behind the options. J Antimicrob Chemother 2010;65: 1094-1099.

3. Daar ES, Tierney C, Fischl MA, et al.: Atazanavir plus ritonavir or efavirenz as part of a 3-drug regimen for initial treatment of HIV-1. Ann Intern Med 2011;154:445-456.

4. Molina JM, Andrade-Villanueva J, Echevarria J, et al.: Oncedaily atazanavir/ritonavir versus twice-daily lopinavir/ ritonavir, each in combination with tenofovir and emtricitabine, for management of antiretroviral-naive HIV-1infected patients: 48 week efficacy and safety results of the CASTLE study. Lancet 2008;372:646-655.

5. Molina JM, Andrade-Villanueva J, Echevarria J, et al.: Once-daily atazanavir/ritonavir compared with twice-daily lopinavir/ritonavir, each in combination with tenofovir and emtricitabine, for management of antiretroviral-naive HIV1-infected patients: 96-week efficacy and safety results of the CASTLE study. J Acquir Immune Defic Syndr 2010;53:323-332.

6. Mallolas J, Podzamczer D, Milinkovic A, et al.: Efficacy and safety of switching from boosted lopinavir to boosted atazanavir in patients with virological suppression receiving a LPV/r-containing HAART: The ATAZIP study. J Acquir Immune Defic Syndr 2009;51:29-36.

7. Johnson M, Grinsztejn B, Rodriguez C, et al.: 96-week comparison of once-daily atazanavir/ritonavir and twice-daily lopinavir/ritonavir in patients with multiple virologic failures. AIDS 2006;20:711-718.

8. Panel on Antiretroviral Guidelines for Adults and Adolescents: Guidelines for the use of antiretroviral agents in HIV1-infected adults and adolescents. Department of Health and Human Services. October 14, 2011; 1-167. http://aidsinfo .nih.gov/contentfiles/AdultandAdolescentGL.pdf. Accessed 17 January 2012.

9. Thompson MA, Aberg JA, Hoy JF, et al.: Antiretroviral treatment of adult HIV infection: 2012 recommendations of the International Antiviral Society-USA panel. JAMA 2012; 308:387-402.

10. European AIDS Clinical Society: Guidelines Version 6.0October 2011. www.europeanaidsclinicalsociety.org/images/ stories/EACS-Pdf/eacsguidelines-v6_english.pdf. Accessed 17 January 2012.

11. Elzi L, Marzolini C, Furrer H, et al.: Treatment modification in human immunodeficiency virus-infected individuals starting combination antiretroviral therapy between 2005 and 2008. Arch Intern Med 2010;170:57-65.

12. Maggiolo F, Leone S, Cologni G, Valenti D, Quinzan G, and Suter F: Persistence on first line HAART is influenced by dosing schedule. Abstracts of the 18th International AIDS Society World AIDS Conference. Vienna, Austria, 2010.

13. Vo TT, Ledergerber B, Keiser O, et al.: Durability and outcome of initial antiretroviral treatments received during 2000-2005 by patients in the Swiss HIV Cohort Study. J Infect Dis 2008;197:1685-1694.

14. Rubio R, Serrano O, Carmena J, et al.: Effect of simplification from protease inhibitors to boosted atazanavir-based regimens in real-life conditions. HIV Med 2010;11:545-553.

15. Pierone G Jr, Mieras J, Martin A, and Urban T: Seven-year follow-up on lopinavir/ritonavir monotherapy. J Int Assoc Physicians AIDS Care (Chic) 2009;8:191-192.

16. Willig JH, Aban I, Nevin CR, et al.: Darunavir outcomes study: Comparative effectiveness of virologic suppression, regimen durability, and discontinuation reasons for threeclass experienced patients at 48 weeks. AIDS Res Hum Retroviruses 2010;26:1279-1285.

17. Cuzin L, Flandre $P$, Pugliese $P$, et al.: Atazanavir in patients with persistent viral replication despite HAART: Results from the French prospective NADIS cohort. HIV Clin Trials 2008;9:147-151.

18. Pugliese P, Cuzin L, Enel P, et al.: [NADIS 2000, development of an electronic medical record for patients infected by HIV, HBV and HCV]. Presse Med 2003;32:299-303.

19. Jansen K, Michalik C, Hahn M, et al.: The patient cohort of the German Competence Network for HIV/AIDS (KompNet): A profile. Eur J Med Res 2009;14:323-331.

20. National Research Council: The Prevention and Treatment of Missing Data in Clinical Trials. Panel on Handling Missing Data in Clinical Trials. Committee on National Statistics, Division of Behavioral and Social Sciences and Education. The National Academies Press, Washington, DC, 2010.

21. Routman JS, Willig JH, Westfall AO, et al.: Comparative efficacy versus effectiveness of initial antiretroviral therapy in clinical trials versus routine care. Clin Infect Dis 2010;50: 574-584.

22. Thompson MA, Aberg JA, Cahn P, et al.: Antiretroviral treatment of adult HIV infection: 2010 recommendations of the International AIDS Society-USA panel. JAMA 2010;304: 321-333.

23. Burgos J, Crespo M, Falcó V, et al.: Simplification to dual antiretroviral therapy including a ritonavir-boosted protease inhibitor in treatment-experienced HIV-1-infected patients. J Antimicrob Chemother 2012;67:2479-2486.

24. Dong BJ, Ward DJ, Chamberlain LA, et al.: Safety and effectiveness of tenofovir/emtricitabine or lamivudine plus ritonavir boosted atazanavir in treatment experienced HIV infected adults at two urban private medical practices. J Antivir Antiretrovir 2012;4:1-5.

25. Squires KE, Johnson M, Yang R, et al.: Comparative gender analysis of the efficacy and safety of atazanavir/ritonavir and lopinavir/ritonavir at 96 weeks in the CASTLE study. J Antimicrob Chemother 2011;66:363-370.

26. Roberts KJ and Mann T: Barriers to antiretroviral medication adherence in HIV-infected women. AIDS Care 2000;12: 377-386.

27. Zhang D, Chando TJ, Everett DW, Patten CJ, Dehal SS, and Humphreys WG: In vitro inhibition of UDP glucuronosyltransferases by atazanavir and other HIV protease inhibitors 
and the relationship of this property to in vivo bilirubin glucuronidation. Drug Metab Dispos 2005;33:1729-1739.

28. Uy J, Hu W, Wirtz V, et al.: Clinical significance of hyperbilirubinemia in the CASTLE study. J Int AIDS Soc 2010;13:P93.

29. Jao J and Wyatt CM: Antiretroviral medications: Adverse effects on the kidney. Adv Chronic Kidney Dis 2010;17:72-82.

30. Rockwood N, Mandalia S, Bower M, Gazzard B, and Nelson M: Ritonavir-boosted atazanavir exposure is associated with an increased rate of renal stones compared with efavirenz, ritonavir-boosted lopinavir and ritonavir-boosted darunavir. AIDS 2011;25:1671-1673.

31. Gandhi M, Ameli N, Bacchetti $P$, et al.: Eligibility criteria for HIV clinical trials and generalizability of results: The gap between published reports and study protocols. AIDS 2005; 19:1885-1896.

32. Phillips AN, Grabar S, Tassie JM, Costagliola D, Lundgren JD, and Egger M: Use of observational databases to evaluate the effectiveness of antiretroviral therapy for HIV infection: Comparison of cohort studies with randomized trials. EuroSIDA, the French Hospital Database on HIV and the Swiss HIV Cohort Study Groups. AIDS 1999;13:2075-2082.

33. Concato J, Shah N, and Horwitz RI: Randomized, controlled trials, observational studies, and the hierarchy of research designs. N Engl J Med 2000;342:1887-1892.

Address correspondence to: Klaus Jansen St. Josef-Klinik, Ruhr-Universität Bochum Clinic for Dermatology, Venerology and Allergology Gudrunstraße 56 44791 Bochum

Germany

E-mail: klaus.jansen@klinikum-bochum.de 